\title{
Introducción \\ Construyendo una Red Chilena para Estudios Socioecológicos a Largo Plazo: Avances, enfoques y relevancia
}

\author{
Introduction \\ Building a Chilean Network for Long-Term Socio-Ecological Research: Advances, \\ perspectives and relevance
}

\author{
CHRISTOPHER B. ANDERSON ${ }^{1,2}{ }^{2}{ }^{*}$, RICARDO ROZZI1, 2, 3, JUAN J. ARMESTO ${ }^{2,4}$ \& JULIO R. GUTIÉRREZ1, 5 \\ ${ }^{1}$ Sub-Antarctic Biocultural Conservation Program, Universidad de Magallanes and University of North Texas, Puerto \\ Williams, Chile \& Denton, Texas, USA \\ 2 Institute of Ecology and Biodiversity, Casilla 653, Santiago, Chile \\ ${ }^{3}$ Department of Philosophy and Religion Studies, University of North Texas, Denton, Texas, USA \\ ${ }^{4}$ Center for Advanced Studies in Ecology and Biodiversity, Departamento de Ecología, Pontificia Universidad Católica de \\ Chile, Santiago, Chile \\ ${ }^{5}$ Departamento de Biología y Centro de Estudios Avanzados en Zonas Áridas, Universidad de La Serena, La Serena, Chile \\ *Autor correspondiente: christopher.anderson@alumni.unc.edu
}

\begin{abstract}
RESUMEN
Desde su inicio formal en 1980, los programas de investigación ecológica a largo plazo (LTER por su sigla en inglés) han servido como un exitoso marco organizacional para crear agendas de investigación y mecanismos de financiamiento que permiten a los científicos abordar fenómenos ecológicos significativos a las escalas en que ellos ocurren. En sus 30 años de existencia, LTER ha expandido su rango geográfico (actualmente la red Internacional de LTER tiene más de 40 países miembros con sitios de estudios en cada uno de los continentes) y focos disciplinarios (principalmente abarcando las ciencias naturales y sociales, llevando a algunos a pedir un cambio de nombre a estudios de investigación socioecológica de largo plazo - LTSER por su sigla en inglés). No obstante, los biomas templados y subantárticos del sur de Sudamérica han carecido de sitios y redes formales de investigación a largo plazo. Sin embargo, numerosos esfuerzos de investigación a largo plazo han existido en Chile y Argentina sin coordinación entre sí. Particularmente, en 2008, el Instituto de Ecología y Biodiversidad puso en marcha el primer esfuerzo sistemático para articular tres sitios existentes (Parque Nacional Fray Jorge - $33^{\circ} \mathrm{S}$, Estación Biológica Senda Darwin - $43^{\circ} \mathrm{S}$, y Parque Etnobotánico Omora - $55^{\circ} \mathrm{S}$ ). Aquí presentamos un número especial de la Revista Chilena de Historia Natural, dedicado a LTSER, con el objetivo de 1) dar una síntesis de algunos de los casos más emblemáticos casos de investigación socioecológica a largo plazo en Chile; 2) demostrar el valor de estos esfuerzos para la integración de la investigación y educación con resultados y procesos sociales, tales como aquellos relacionados con la toma de decisiones; y 3) ofrecer la perspectiva de una amplia gama de participantes involucrados en estas iniciativas, incluyendo a estudiantes de postgrado y programas asociados desde Iberoamérica y Norteamérica. Es nuestro deseo que estos trabajos compilados contribuyan a la consolidación de la aproximación de LTSER en el sur de Sudamérica dentro de la comunidad académica y también para una mayor integración entre la academia y la sociedad.
\end{abstract}

Palabras clave: LTER, LTSER, subantártico, Sudamérica, templado.

\begin{abstract}
Since their formal inception in 1980, long-term ecological research (LTER) programs have served as a successful organizing framework to create research agendas and funding mechanisms that allow scientists to address meaningful ecological phenomena at the scales they occur. In its 30 years of existence, LTER has expanded its geographic range (currently the International LTER network has more than 40 country members with sites on every continent) and disciplinary foci (principally encompassing the natural and social sciences and leading some to call for a name change to long-term socio-ecological research - LTSER). Nonetheless, the temperate and subantarctic biomes of southern South America have lacked formalized longterm research sites and networks. Yet, at the same time, numerous uncoordinated long-term research efforts exist in both Chile and Argentina, and in 2008, the Institute of Ecology and Biodiversity launched Chile's first concerted effort to link three existing sites (Fray Jorge Forest National Park - 33 ${ }^{\circ}$ S, Senda Darwin Biological
\end{abstract}


Station - $43^{\circ} \mathrm{S}$, and Omora Ethnobotanical Park - $55^{\circ} \mathrm{S}$ ). Here, we present a special feature of the Revista Chilena de Historia Natural, dedicated to LTSER, with the aim of 1) providing a synthesis of some of the most emblematic cases of long-term socio-ecological research in Chile; 2) demonstrating the value of these efforts for the integration of research, education and social outcomes, such as decision making; and 3) offering the perspective of a broad array of participants involved in these initiatives, including graduate students and associated programs from Ibero-America and North America. It is our hope that these compiled works will contribute to the consolidation of the LTSER approach in southern South America both within the academic community and also to better link academia and society.

Key words: LTER, LTSER, South America, subantarctic, temperate.

\section{ANTECEDENTES DE LTER Y LTSER}

El término "investigación ecológica a largo plazo" (LTER, por su sigla en inglés) fue acuñado por el programa homónimo de la Fundación Nacional de la Ciencia de Estados Unidos (del inglés, US National Science Foundation), establecido en 1980. Esta primera red LTER formalizó varias décadas de trabajo de un numeroso grupo de ecólogos, quienes fundamentaban la necesidad institucionalizar una red de investigación y un mecanismo de financiamiento a largo plazo. Esta innovadora plataforma de investigación les permitiría abarcar las grandes escalas temporales y geográficas en que ocurren muchos fenómenos ecológicos, que no son abordables mediante una colección de proyectos discretos y de corto plazo (Callahan 1984, Franklin et al. 1990).

Desde su conceptualización y creación, el programa LTER ha crecido tanto en el sentido geográfico como en el temático. Durante las primeras dos décadas, el programa LTER creció desde cinco sitios con un presupuesto anual de $\$ 1.2$ millones de dólares (USD) hasta una red que incluyó veinticuatro sitios ecológicamente diversos con un presupuesto directo de $\$ 17.8$ millones USD (año fiscal 2002), involucrando a más de mil científicos y estudiantes que generaron aproximadamente \$ 44 millones USD en investigación relacionada con LTER en la red completa (NSF 2002). Temáticamente, la primera década de LTER se focalizó en la recolección y análisis de datos en cinco áreas principales de investigación a largo plazo: producción primaria, flujo de nutrientes, estructuras tróficas, perturbaciones, y acumulación y descomposición de materia orgánica.

Durante la segunda década, el programa LTER amplió su ámbito de investigación, incluyendo las influencias antrópicas en los sistemas ecológicos. Esta adaptación de la agenda de investigación surgió de la revisión de los primeros diez años de experiencias en LTER por la NSF, que enfatizó la necesidad de incluir patrones y procesos a gran escala y comparaciones entre sitios (NSF 2002). En consecuencia, el programa LTER promovió la interdisciplinaridad, la cooperación científica interinstitucional e internacional, y en la actualidad aproximadamente 40 países están asociados a los programas de la Red Internacional LTER (ILTER por su sigla en inglés).

Durante la última década, la iniciativa mundial de LTER ha expresado explícitamente su propósito de articular disciplinas relevantes que van más allá de la ecología, dentro de la academia (e.g., antropología, sociología, historia ambiental, entre otros; véase Redman et al. 1998) y también más allá de la "torre de marfil”, integrando los esfuerzos de investigación con ámbitos de relevancia social (e.g., educación, extensión, desarrollo de políticas públicas; véase Hobbie et al. 2003). Al mismo tiempo, hoy en día varias redes de investigación ecológica a largo plazo, tales como la Red del Observatorio Ecológico Nacional (NEON por su sigla en inglés) en Estados Unidos de América, están en la vanguardia mediante la incorporación de tecnologías de última generación, tales como sensores remotos para registros continuos de variables físicas y biológicas y otros métodos para trabajo en red y monitoreos, para lograr escalas de resolución más fina que permitan comprender los procesos ecosistémicos fundamentales a escalas continentales (Keller et al. 2008).

De esta forma, durante sus tres décadas de existencia, la agenda LTER se ha ido transformando desde un enfoque centrado en áreas silvestres, muchas veces áreas protegidas, hacia una perspectiva cada vez más 
compleja e integradora que incluye ciudades, paisajes rurales/agrícolas y otros factores sociales como parte de sistemas acoplados humanos-naturaleza. Esta perspectiva más amplia sobre los fenómenos ecológicos ha conducido a adoptar el nombre de "investigación socioecológica a largo plazo" (Long-Term Socio-Ecological Research LTSER por su sigla en inglés), con un enfoque que incluye variables socioeconómicas (Haberl et al. 2006). Recientemente, por ejemplo, US LTER propuso el modelo "Ciencia Integrada para la Sociedad y el Ambiente” (Integrated Science for Society and the Environment: ISSE) que combina dimensiones sociales $y$ ecológicas de los ecosistemas a través de un enfoque basado en la comprensión de umbrales y ciclos de retroalimentación entre las percepciones y conductas humanas, la estructura y función de los ecosistemas, y el suministro de bienes y servicios ecosistémicos (Collins et al. 2007). Actualmente, la red ILTER está llevando a cabo un proyecto piloto (véase Maass et al. 2010, en este número) que intenta aplicar este marco conceptual en tantas redes nacionales y regionales como sea posible, a fin de probar su utilidad para comparar procesos a través de biomas y fronteras políticas (Bourgeron et al. datos no publicados). No obstante, hasta la fecha, las aproximaciones para implementar LTSER están centradas principalmente en la integración de las ciencias naturales y sociales, en particular la economía (pero véase Swanson et al. 2008, para un caso de integración entre científicos y escritores).

Como afirmaron Costanza et al. (1997), "los servicios ecosistémicos no son completamente 'capturados' por los mercados, o adecuadamente cuantificados en términos comparables con servicios económicos y capital manufacturado"; por lo tanto, hay una necesidad crítica para desarrollar una valoración monetaria más completa. Sin embargo, los valores y metodologías económicas no incluyen todas las dimensiones sociales. Consecuentemente, existe aún una necesidad persistente y significativa por incluir en forma más holística las múltiples facetas de la cultura, e incorporar metodologías filosóficas y de ciencias sociales que permitan abarcar la amplia gama de valores y prácticas culturales en la investigación ecosistémica a largo plazo, y contribuir de esta manera a la conservación de la diversidad biológica y cultural (Rozzi et al. 2008).

Hasta ahora existen aproximadamente 40 programas nacionales formales que son miembros de la red ILTER. Sin embargo, dentro de la cobertura de la red ILTER encontramos una notoria carencia de programas y sitios de investigación consolidados, especialmente en un rango latitudinal, el área entre $40-60^{\circ} \mathrm{S}$, que incluye, además de la estepa patagónica, al bioma templado-subantártico ambos compartidos entre Argentina y Chile en el sur de América (Rozzi et al. datos no publicados). Esta región presenta características singulares que la hacen relevante a nivel mundial para la investigación socioecológica a largo plazo, especialmente el análisis de procesos vinculados al cambio global. Por ejemplo, a lo largo de dicha extensión latitudinal encontramos un marcado gradiente de variables relevantes para experimentos ecosistémicos, incluyendo temperatura, fotoperíodo, precipitación, regímenes de perturbación, impacto antrópico, exposición a UV debido al adelgazamiento de la capa de ozono, topografía y ensambles de especies con un alto grado de endemismo (Lawford et al. 1996). Esta área incluye también la mayor extensión de bosques templados (Armesto et al. 1996), humedales, formaciones de tundra y turberas (Arroyo et al. 2006) y campos de hielo ( $\sin$ considerar la Antártida) en el hemisferio sur. Es también una de las escasa zonas del planeta que no han estado sujetas a una deposición atmosférica crónica y creciente de nitrógeno, de origen antropogénico, constituyéndose en un referente mundial para estudios ecosistémicos de condiciones biogeoquímicas del planeta previas a la Revolución Industrial (Galloway et al. 2004, Armesto et al. 2009). Desde el punto de vista de la diversidad cultural, el extremo sur de Sudamérica alberga los grupos étnicos y la familia lingüística más australes del mundo; además, dos de las lenguas fueguinas están en peligro, debido a que menos de diez personas hablan hoy fluidamente las lenguas kaweshkar y yagán (Rozzi et al. 2003)

Con respecto al cambio climático global, se espera que la región austral de Sudamérica experimente tendencias contrastantes en 
cambios de temperatura y precipitación en sus latitudes medias y altas (CONAMA 2006). Esta región cuenta con registros detallados y únicos de cambios climáticos y bióticos que han ocurrido durante los últimos milenios a lo largo de sus gradientes topográficos y latitudinales; estos registros proveen información paleobiológica que puede ser crucial para entender los patrones globales de variabilidad climática (Latorre et al. 2007). Por lo tanto, la ausencia de sitios de investigación a largo plazo establecidos en esta área, y su conectividad con redes científicas nacionales e internacionales, constituye una preocupación no solo para el desarrollo de una ciencia predictiva de primer nivel en Chile y Argentina, sino también para lograr llenar este vacío de registros que son esenciales para la comprensión de patrones globales y procesos terrestres y marinos. Asimismo, estos estudios pueden proveer la información científica necesaria para recomendar respuestas que la sociedad humana deberá dar frente a cambios esperados en el clima y los servicios ecosistémicos. Para tener éxito en este nuevo énfasis en una red LTSER coordinada en el sur de Sudamérica será necesario superar obstáculos significativos en cuanto a limitaciones de infraestructura, coordinación, conformación de redes de comunicación, personal y financiamiento. Los procesos de implementación de esta red austral están todavía en curso (Anderson et al. 2008).

\section{PRESENTACION DE ESTE TEMA ESPECIAL}

Este Tema Especial de la Revista Chilena de Historia Natural (RChHN), dedicado a LTSER, documenta un avance con respecto a convocatorias iniciales para formalizar una estrategia nacional de investigación ecológica a largo plazo en Chile (Armesto 1990, 1995). También es un paso para formalizar los vínculos de la ciencia chilena y de una región subestudiada del hemisferio sur con iniciativas internacionales para monitorear y entender los sistemas planetarios, como ILTER, NEON, GLEON (http:// www.ilternet.ed, http://www.neoninc.org, http://www.gleonrcn.org), entre otros. Con este objetivo en mente, se presenta esta colección de artículos que en su mayoría resultaron del taller titulado "Enfrentando el cambio global con una red de sitios de estudios socio-ecológicos a largo plazo en el sur de las Américas”, organizado en junio de 2008 en el extremo austral de América, en la Región de Magallanes y Antártica Chilena.

Financiado por el Programa de Redes Formales de la Iniciativa Científica Milenio (MIDEPLAN) y el Programa de Financiamiento Basal (CONICYT), con apoyo del Hispanic Global Initiative de la University of North Texas, este encuentro tuvo como misión principal el lanzamiento del primer esfuerzo chileno para articular tres sitios LTSER, coordinados por el Instituto de Ecología y Biodiversidad (http://www.iebchile.cl), y en este proceso, congregar a investigadores vinculados con la investigación a largo plazo en Chile y conocer experiencias complementarias de redes LTER nacientes y avanzadas en Argentina, México, España, y EE.UU.

Para ampliar las perspectivas incluidas en este Tema Especial, los organizadores hemos invitado contribuciones de varias otras iniciativas importantes en investigación a largo plazo en Chile, que incorporan distintos tipos de ecosistemas, así como también programas asociados de otros países de Ibero-América que enriquecen el diálogo internacional. Aunque este esfuerzo no representa una lista exhaustiva de todos los estudios a largo plazo realizados hasta la fecha en Chile, establecidos de manera independiente por centros académicos y estaciones de campo privadas, es nuestro propósito que el presente número de $\mathrm{RChHN}$ facilite un primer paso de integración y haga un llamado a la coordinación de dichos esfuerzos dentro de la iniciativa LTSER-Chile. En particular, la reflexión y el debate durante la inauguración de la primera Red LTSER en Chile en Puerto Williams en junio de 2008 se centró en los siguientes tres objetivos:

(1) Lograr un acuerdo social, académico y político sobre la aproximación LTSER. La inauguración formal de la naciente Red de Sitios de Estudios Socioecológicos a Largo Plazo en Chile permitió que los participantes de los tres sitios coordinados por IEB, y sus colaboradores, presentaran sus agendas de investigación a las autoridades locales, regionales, nacionales e internacionales y 
demostraran su capacidad de sustentar la investigación práctica y teórica para el avance, tanto de los intereses académicos, como de la integración de la ciencia y la sociedad en ecosistemas cruciales para el país.

(2) Utilizar el concepto LTSER para establecer colaboraciones internacionales efectivas a largo plazo. Por razones logísticas y financieras, la red inicial se ha conformado con tres sitios de estudio en Chile (Fig. 1): Parque Nacional Bosque Fray Jorge $\left(33^{\circ} \mathrm{S}\right)$, Estación Biológica Senda Darwin $\left(43^{\circ} \mathrm{S}\right)$ y Parque Etnobotánico Omora $\left(55^{\circ} \mathrm{S}\right)$. Estos sitios tienen vínculos preliminares, pero formales, con tres redes internacionales: la Red Internacional de Investigación Ecológica a Largo Plazo (ILTER), la Red Ibero-Americana de Reservas de Biosfera (Ibero-MaB), y el Center for Embedded Network Sensing de la Universidad de California Los Angeles (CENSUCLA).

(3) Ayudar a institucionalizar los esfuerzos LTSER como un paradigma de investigación transdisciplinaria. La agenda promovida por la red nacional intenta fortalecer las bases y el diseño para una aproximación que es interdisciplinaria e interinstitucional. Para ello, trabaja en la creación de programas de educación y cursos de campo internacionales que integran las ciencias ecológicas, la filosofía ambiental y otras aproximaciones para entender el entorno natural a fin de generar soluciones viables y novedosas para los problemas medioambientales y sociales interrelacionados. Además, la red crece y se construye sobre la base de las relaciones existentes de los sitios individuales que van más allá del mundo académico e incluyen al sector público y privado.

El taller en 2008 reunió a más de veinticinco científicos de cinco países a largo de América, y también de España, y convocó más de veinticinco autoridades del nivel local, regional, nacional e internacional, incluyendo el evento inaugural en Puerto Williams, Chile (Fig. 2), que fue presidido por la intendenta regional Sra. Eugenia Mancilla (Fig. 3). Además, la reunión científica fue organizada en coincidencia con el curso de conservación biocultural de campo internacional titulado "Siguiendo las huellas de Darwin" (http:// www.chile.unt.edu/projects/tdp/tdp- index.html), uno de los tres cursos de campo que se llevan a cabo anualmente por la Red LTSER del IEB (http://www.ieb-chile.cl/ ltser/curso_campo.php). A partir de este paso inicial, se ha reforzado un diálogo con ILTER, USLTER y otros colaboradores nacionales e internacionales en el diseño de una red que nos permita enfrentar los impactos del cambio global en los ecosistemas y la sociedad.

El objetivo de este Tema Especial en el presente número de $\mathrm{RChHN}$ es colaborar en el proceso de comunicación y refinamiento de la estrategia LTSER por parte de científicos y administradores de la ciencia en Chile y otros países de América. En este número, hemos procurado: (a) resumir varios de los casos más emblemáticos de investigación a largo plazo que operan en Chile; (b) demostrar el valor de tales esfuerzos para la integración de la investigación, la educación y la toma de decisiones; y (c) ofrecer las perspectivas de una amplia gama de actores, incluyendo estudiantes de postgrado y programas asociados dentro de Iberoamérica y Norteamérica. Esperamos que estos trabajos contribuyan a guiar el proceso del establecimiento de LTSER en Chile y, al mismo tiempo, estimular la formación de una nueva generación de profesionales capaces de trabajar en la interfase ciencia-sociedad, y así abordar de manera más integral los futuros desafíos sobre el manejo de ecosistemas y la conservación en un mundo cambiante (Pielke et al. 2007). Esto requiere un entendimiento de los cambios que ocurren en sistemas naturales y antrópicos acoplados. Para ello, es necesaria una perspectiva a largo plazo que haga posible anticipar y modelar escenarios de cambio global, que constituyen áreas de incertidumbre del desarrollo socioeconómico, especialmente para países como Chile que han dependido históricamente en gran medida de la explotación de su capital natural. En consecuencia, los documentos incluidos en el presente Tema Especial deben ser considerados como una fuente de información relevante no solo para la comunidad académica, sino también para contribuir al avance de las políticas públicas y la toma de decisiones relacionadas con el papel de la ciencia y la investigación en el desarrollo social y el bienestar de Chile. 


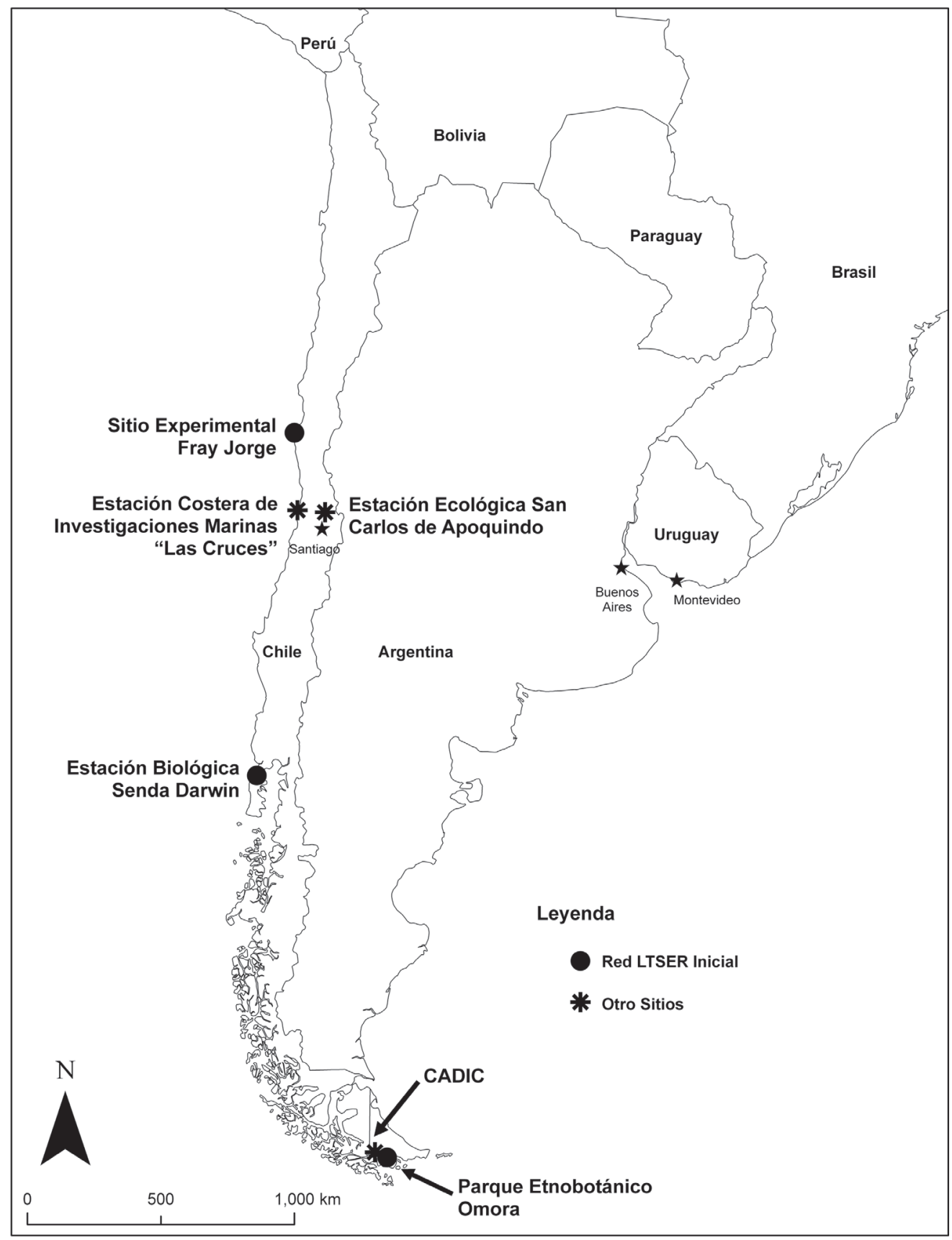

Fig. 1: La primera Red Chilena de Sitios de Estudios Socio-Ecológicos a Largo Plazo (LTSER), coordinada por el Instituto de Ecología y Biodiversidad, incluye tres sitios preliminares (círculos sólidos) que abarcan el ámbito de distribución latitudinal de los bosques en Chile. Programas complementarios (indicados con cruces) fueron invitados para contribuir a esta edición especial y enriquecer el diálogo de LTSER en Chile y América Latina, a la vez que demostrar su potencial de crecimiento en etapas futuras. (Mapa elaborado en el UNT Center for Spatial Analysis \& Mapping, P. Palacios).

The initial Chilean Long-Term Socio-Ecological Research Network (LTSER), coordinated by the Institute of Ecology and Biodiversity, includes three preliminary sites (solid circles) that span the latitudinal range of forests in Chile. Complementary programs (denoted with crosses) were invited to contribute to this special edition to enrich to dialogue on LTSER in Chile and Latin America and demonstrate its potential growth in the future stages. (Map elaborated in UNT Center for Spatial Analysis \& Mapping, P. Palacios). 
ORGANIZACIÓN DE ESTE TEMA ESPECIAL

Para comunicar los objetivos anteriores de forma más clara y precisa, hemos organizado este Tema Especial en cuatro secciones que se describen a continuación.

\section{La relevancia internacional de los pasos preli- minares en Chile hacia LTSER}

Prólogo: Dr. Terry Parr actualmente se desempeña como Director del Comité Ejecutivo de International Long-Term
Ecological Research (ILTER) y en este rol coordina a las más de 40 redes que son miembros. También, es el Director de ALTERNet, una red de investigación a largo plazo sobre biodiversidad y ecosistemas con veintidós socios europeos en diecisiete países, y además participa con varias funciones de ESFRI/European LifeWatch Program y la iniciativa global Diversitas. En su prólogo para esta edición especial, relata su comprensión del contexto mundial de la investigación a largo plazo y su relación con los esfuerzos nacientes en Chile y el Cono Sur.

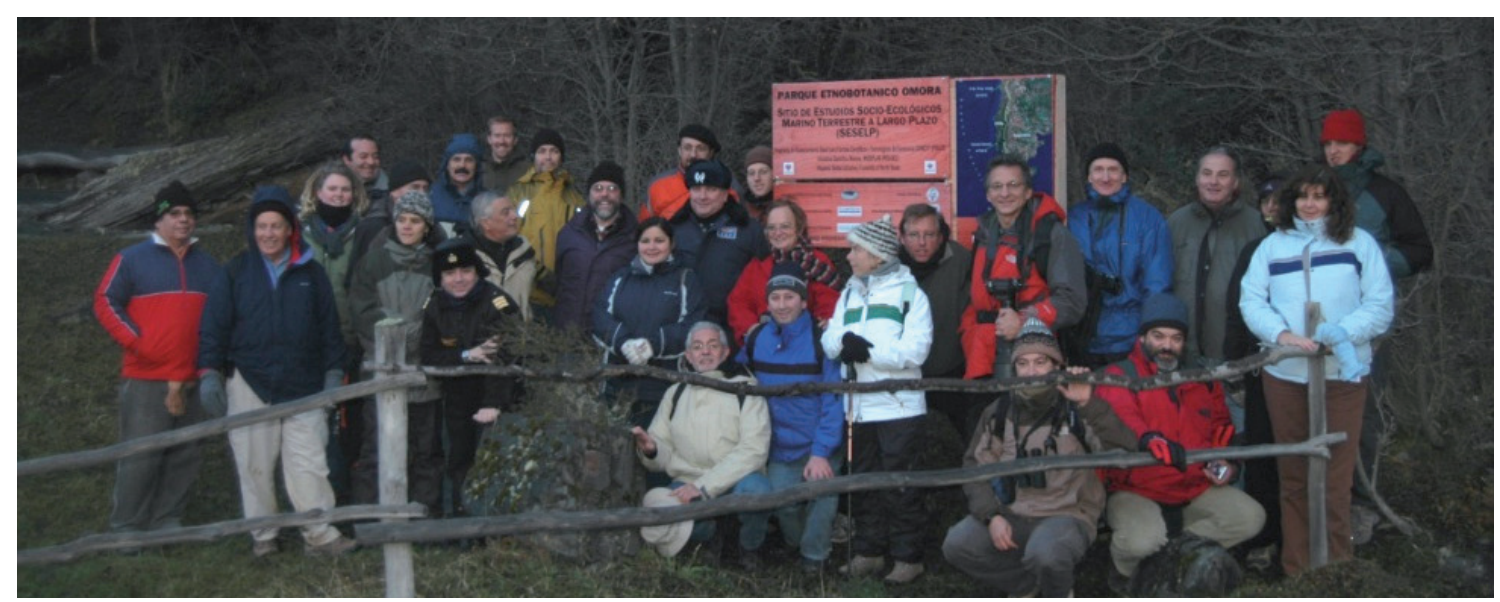

Fig. 2: La inauguración de la primera Red LTSER-Chile en el Parque Etnobotánico Omora durante junio de 2008 (de izquierda a derecha- ${ }^{\text {er }}$ plano: Julio Gutiérrez (ULS-IEB), Phil Rundel (UCLAUSA), Comandante Francisco Abrego (Armada de Chile), Sergio Guevara (Ibero-MaB), Andrés Mansilla (UMAG-IEB), Dinah Arnett (Embajada de EE.UU. en Chile), Ricardo Díaz-Delgado (CSIC-España), Francisco Squeo (ULS-IEB), Graciela Alcina (Tierra del Fuego, Argentina); $2^{\circ}$ plano: Kelli Moses (OSARA-UNT), James Kennedy (UNT-USA), Aurora Gaxiola (IEB), Juan Armesto (PUC-IEB), Yanet Medina (UMAG-IEB), Mary Kalin (UCh-IEB), Robert Frodeman (UNTUSA), Eduard Mueller (UCI-Costa Rica), Bron Taylor (UF-USA), Alejandro Rojo-Vivot (AVINAPatagonia); $3^{\text {er }}$ plano: Cristián Guerrero (Omora), Manuel Maass (UNAM-México), Eric Graham (UCLA-USA), Adrian Schiavini (CADIC-Argentina), Ricardo Rozzi (UNT-UMAG-IEB), General Luis Ili (Fuerza Aérea de Chile), Guillermo Martínez Pastur (CADIC-Argentina), Christopher Anderson (UMAG-IEB-UNT). No se encuentran en la foto: Carolina Henríquez (IEB), J. Britt Holbrook (UNT-USA), Ione Hunt Von Herbing (UNT-USA), Francisca Massardo (UMAG-IEB), Rodrigo Vásquez (UCh-IEB).

The inauguration of the first LTSER-Chile Network at the Omora Ethnobotanical Park in June 2008 (from left to right - $1^{\text {st }}$ row: Julio Gutiérrez (ULS-IEB), Phil Rundel (UCLA-USA), Comandant Francisco Abrego (Chilean Navy), Sergio Guevara (Ibero-MaB), Andrés Mansilla (UMAG-IEB), Dinah Arnett (US Embassy in Chile), Ricardo DíazDelgado (CSIC-Spain), Francisco Squeo (ULS-IEB), Graciela Alcina (Tierra del Fuego, Argentina); $2^{\text {nd }}$ row: Kelli Moses (OSARA-UNT), James Kennedy (UNT-USA), Aurora Gaxiola (IEB), Juan Armesto (PUC-IEB), Yanet Medina (UMAG-IEB), Mary Kalin (UCh-IEB), Robert Frodeman (UNT), Eduard Mueller (UCI-Costa Rica), Bron Taylor (UF-USA), Alejandro Rojo-Vivot (AVINA-Patagonia); $3^{\text {rd }}$ row: Cristián Guerrero (Omora), Manuel Maass (UNAMMéxico), Eric Graham (UCLA-USA), Adrian Schiavini (CADIC-Argentina), Ricardo Rozzi (UNT-UMAG-IEB), General Luis Ili (Chilean Air Force), Guillermo Martínez Pastur (CADIC-Argentina), Christopher Anderson (UMAG-IEBUNT). Not pictured: Carolina Henríquez (IEB), J. Britt Holbrook (UNT-USA), Ione Hunt Von Herbing (UNT-USA), Francisca Massardo (UMAG-IEB), Rodrigo Vásquez (UCh-IEB). 


\section{Integración de la investigación, la educación y la toma de decisiones}

Estévez y colaboradores conforman un grupo de estudiantes de postgrado de diversas disciplinas (filosofía, biología y conservación), de dos universidades regionales chilenas (Universidad de La Serena y Universidad de Magallanes) y una universidad estadounidense (University of North Texas). En su trabajo presentan una visión crítica de los programas de postgrado en conservación en universidades chilenas y ofrecen opiniones sobre qué dirección deberían tomar las iniciativas educativas interdisciplinarias para producir una generación de estudiantes capaces de abordar investigaciones sobre las dimensiones integradas sociales-ecológicas.

Rozzi y colaboradores desarrollan su trabajo basado en la experiencia de los primeros diez años del programa de investigación, educación y conservación biocultural que se conduce en el Parque Etnobotánico Omora, en Cabo de Hornos, el último confín de América. Este artículo presenta una metodología que consiste en un ciclo de cuatro pasos interrelacionados de (1) investigación ecológica y filosófica, (2) comunicación metafórica, (3) filosofía ambiental de campo y actividades educativas y de ecoturismo guiadas por ecólogos, y (4) conservación in situ. La aproximación "biocultural" propuesta por Omora ha sido desarrollada en conjunto con los programas de posgrado en conservación, filosofía y ecología que llevan a cabo actividades en el Parque Omora, particularmente bajo la coordinación del Programa de Magíster en Ciencias de la Universidad de Magallanes en colaboración con el Instituto de Ecología y Biodiversidad (IEB) y la University of North Texas (UNT).

\section{Programas significativos de LT(S)ER en Chile}

Gutiérrez y colaboradores resumen investigación ecológica experimental a largo plazo que se lleva a cabo en el Parque Nacional Bosque Fray Jorge, norte-centro de Chile, y que es una de las más importantes no solo en Chile y Sudamérica, sino también en todo el hemisferio sur. Su proyecto utiliza un

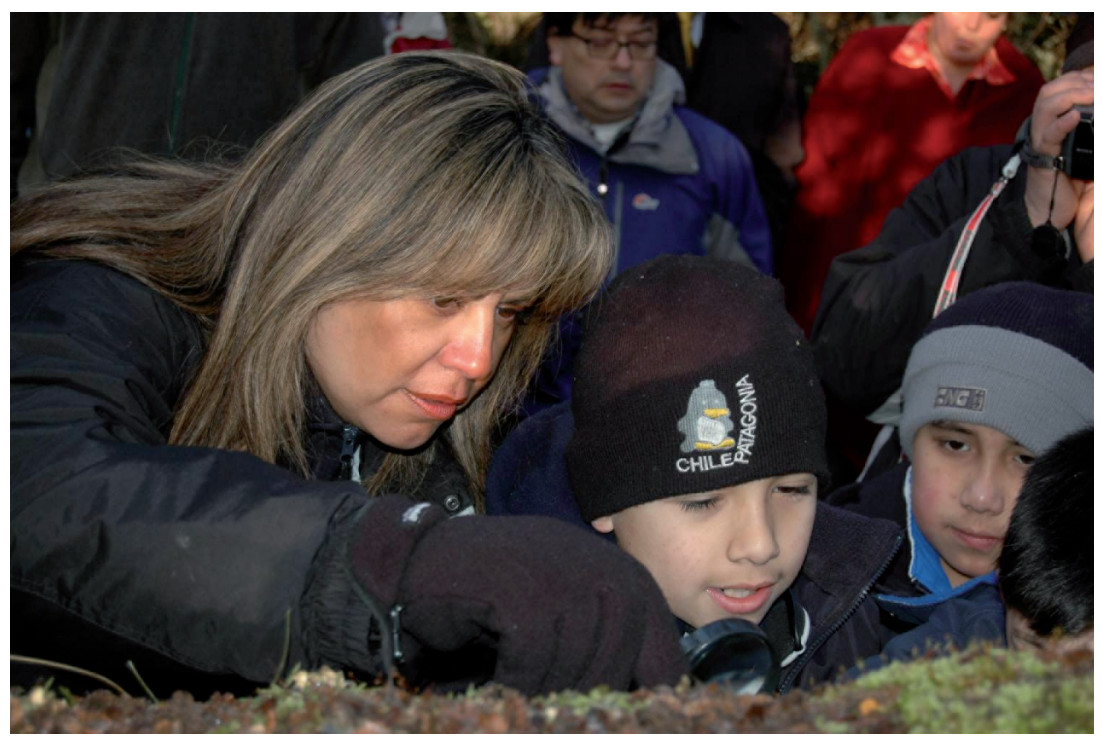

Fig. 3: La intendenta de la Región de Magallanes y Antártica Chilena Sra. Eugenia Mancilla durante su visita al Parque Etnobotánico Omora con alumnos del liceo de Puerto Williams, en la inauguración de la Red Chilena de Sitios de Estudios Socio-Ecológicos a Largo Plazo, coordinada por el Instituto de Ecología y Biodiversidad (IEB), en junio 2008.

Regional Intendenta Ms. Eugenia Mancilla visits the Omora Ethnobotanical Park with school children from Puerto Williams as part of the inauguration of the Chilean Long-Term Socio-Ecological Research Network, coordinated by the Institute of Ecology and Biodiversity (IEB), in June 2008. 
sistema experimental a gran escala que incluye veinte parcelas de 0.56 ha de exclusión selectiva de vertebrados (predadores y herbívoros). En su conjunto, esta aproximación y el compromiso a largo plazo de este equipo multinacional ha permitido detectar patrones en las dinámicas de las comunidades bióticas en ecosistemas áridos, al mismo tiempo que el impacto de la variabilidad climática, como los eventos El Niño, sobre la dinámica de las plantas y su efecto cascada sobre los pequeños mamíferos y sus depredadores (zorros y aves rapaces).

Pavez y colaboradores utilizan datos provenientes de uno de los sitios más estudiados en Chile, ubicado en la Estación de Investigaciones Ecológicas Mediterráneas en San Carlos de Apoquindo, para demostrar el uso de bases de datos a largo plazo para detectar cambios en relaciones ecológicas sutiles, tales como las interacciones tróficas y cómo estas son afectadas por distintos usos de la tierra. De no contar con estos datos de largo plazo, tales dinámicas de las comunidades bióticas podrían pasar "desapercibidas".

Carmona y colaboradores documentan la investigación a largo plazo en los ecosistemas de turbera y bosque, principalmente en la Estación Biológica Senda Darwin en la isla grande de Chiloé. Sus estudios tienen múltiples objetivos, desde la comprensión de la demografía y dinámica poblacional de especies clave de aves, mamíferos y plantas hasta la restauración de especies amenazadas y la conducción de experimentos de campo a largo plazo sobre los efectos del cambio climático global en los procesos de los ecosistemas forestales nativos. Los resultados de estos estudios son utilizados para mejorar las formas de manejo, la conservación de la biodiversidad y el bienestar humano en el entorno rural de la zona del archipiélago de Chiloé.

Navarrete y colaboradores enriquecen el diálogo con la presentación del caso de la Estación Costera de Investigación Marina en Las Cruces, en el litoral central de Chile, donde una pequeña área experimental, excluida de la pesca desde el año 1982, ha adquirido cada vez más importancia hasta llegar a ser uno de los sitios más intensamente estudiados sobre ecología marina-costera en el sur de Sudamérica. De la misma forma, los estudios a largo plazo de los investigadores asociados a dicha estación han permitido que su trabajo sea aplicado directamente sobre las políticas pesqueras y el manejo de los recursos naturales en Chile.

\section{Perspectivas de programas asociados en Ibe- roamérica}

Martínez-Pastur y colaboradores sintetizan datos de cuarenta años de estudio de parcelas forestales experimentales en la Patagonia sur. $\mathrm{Su}$ trabajo efectivamente "redescubre" y destaca una plataforma de investigación previamente olvidada y una serie de recursos subutilizados hasta la fecha, y también ilustra la necesidad fundamental de llamar al diálogo entre investigadores chilenos y argentinos para lograr una comunicación más eficaz sobre ecosistemas compartidos.

Maass y colaboradores utilizan un enfoque comparativo, presentando los casos de la creación de los programas de investigación a largo plazo en México y España. Posteriormente, amplían la discusión utilizando como ejemplo el marco conceptual del ISSE para el estudio de los servicios ecosistémicos, promovido por ILTER con el fin de poner a prueba su utilidad para abordar temáticas ecológicas y sociales relevantes en dos redes cuyos contextos culturales, políticos y económicos son distintos.

Para concluir, Franklin \& Swanson ofrecen una revisión de las estrategias y aportes de la investigación a largo plazo en los ecosistemas forestales de América del Norte al conocimiento científico y cómo estas iniciativas han influido sobre la toma de decisiones que ha afectado a la conservación y al desarrollo de prácticas sustentables de manejo forestal en Norteamérica. Estas perspectivas luego se extrapolan al más amplio significado de los programas LTER y LTSER, para ayudar a mejorar las iniciativas emergentes en los ecosistemas templadosubantárticos del sur de América.

En resumen, este Tema Especial de la Revista Chilena de Historia Natural es un avance importante en un proceso continuo para llevar a cabo mejores y más relevantes investigaciones a largo plazo sobre los sistemas socioecológicos de las ecorregiones templadas y subantárticas en el sur de 
América. El éxito en la implementación de LTSER en Chile requiere de compromisos a largo plazo de financiamiento, infraestructura y apoyo institucional, pero quizás lo más importante para este tipo de iniciativas sea una visión compartida por parte de individuos dedicados a superar los obstáculos (Strayer et al. 1986). Esta comunidad debe asumir la tarea de crear vínculos a largo plazo entre investigadores, la comunidad local y la sociedad en general (Anderson et al. 2008). Es nuestro deseo que este Tema Especial contribuya a fortalecer el esfuerzo LTSER en Chile y el Cono Sur, y acrecentar y formalizar los vínculos entre la comunidad chilena de investigación y los esfuerzos LTSER a nivel internacional. Esta colaboración es esencial para confrontar los grandes desafíos sociales y ecológicos a nivel regional y global del siglo XXI.

\section{MATERIAL COMPLEMENTARIO}

La versión en inglés de este artículo está disponible como Material Complementario online en http://rchn.biologiachile.cl/ suppmat/2010/1/MC_Anderson_et_al_2010.pdf

\section{AGRADECIMIENTOS}

El comité editorial agradece a Dr. Patricio Camus por su apoyo y colaboración en la preparación de este Tema Especial. Asimismo, agradecemos a todas las autoridades, administradores, investigadores y estudiantes que participaron con sus comentarios valiosos y generosos en el taller "Enfrentando el cambio global con una red de sitios de estudios socioecológicos a largo plazo en el sur de América”. El apoyo institucional ha sido proveído por la Universidad de Magallanes, la University of North Texas y el Instituto de Ecología y Biodiversidad, además de la Iniciativa Científica Milenio (MIDEPLAN), a través de un Proyecto de Redes Formales de R. Rozzi, J. Armesto y C.B. Anderson y Proyecto P05-002 y por el Programa de Financiamiento Basal (CONICYT, Proyecto PFB-23). JJA agradece a Dr. G. Likens y los científicos de la Cary Institute of Ecosystem Studies, Nueva York, USA, por inspirar las primeras ideas sobre estudios a largo plazo en Chile.
Reconocemos también el apoyo valioso de los revisores de manuscritos en este Tema Especial y comentarios constructivos de Alexandria Poole (UNT) a esta Introducción. Para las traducciones al castellano se contó con la colaboración de estudiantes de doctorado financiados para trabajar en el Parque Omora a través del Programa de Conservación Biocultural Subantártica y su proyecto International Research Experience for Students (US National Science Foundation OISE: 0854350), incluyendo Christopher Cheleuitte (Texas A\&M University), Deborah Viera (University of North Texas) y Alejandro Valenzuela (Universidad de Buenos Aires), y también con la colaboración de Dra. Silvina Ippi (Universidad de Chile), Jaime Ojeda (UMAG) y J. Cristóbal Pizarro (UMAG). Las traducciones luego fueron revisadas por los Drs. Guillermo Martínez-Pastur (CADICCONICET), Vanessa Lencinas (CADICCONICET) y Martín Carmona (IEB).

\section{LITERATURA CITADA}

ARMESTO JJ (1990) Estudio a largo plazo: Una prioridad para la investigación ecológica de hoy. Revista Chilena de Historia Natural 63: 7-9.

ARMESTO JJ (1995) Fundamentos y necesidades para un programa de estudios de largo plazo de ecología en Chile. Revista Chilena de Historia Natural 68: 5-11.

ARMESTO JJ, C VILLAGRAN \& MTK ARROYO (eds) (1996) Ecología de los bosques nativos de Chile Editorial Universitaria, Santiago, Chile.

ARMESTO JJ, C SMITH-RAMIREZ, MR CARMONA, JL CELIS-DIEZ, IA DIAZ et al. (2009) Old-growth temperate rainforests of South America: Conservation, plant-animal interactions, and baseline biogeochemical processes. En: Wirth C (ed) Old-growth forests Vol. 207: 367-390. Springer-Verlag, Berlin.

ARROYO MTK, P PLISCOFF, M MIHOC \& $\mathrm{M}$ ARROYO-KALIN (2005) The Magellanic moorland. En: Fraser LH \& PA Keddy (eds) The world's largest wetlands: Ecology and conservation: 424-445. Cambridge University Press, Cambridge, UK.

CALLAHAN JT (1984) Long-term ecological research. BioScience 34: 363-367.

COLLINS SL, SM SWINTON, CW ANDERSON, TL GRAGSON, NB GRIMM et al. (2007) Integrated science for society and environment: A strategic research initiative. Albuquerque, Long-Term Ecological Research Network, Publication No. 23. (en línea) URL: http://www.lternet.edu.

COSTANZA R, R D'ARGE, R DE GROOT, S FARBER, M GRASSO et al. (1997) The value of the world's ecosystem services and natural capital. Nature 387: 253-260. 
CONAMA (2006) Estudio de la variabilidad climática en Chile para el siglo XXI. Informe final. Corporación Nacional del Medio Ambiente, Santiago, Chile.

FRANKLIN JF, CS BLEDSOE \& JT CALLAHAN (1990) Contributions of the long-term ecological research program. BioScience 40: 509-523.

GALLOWAY JN, FJ DENTENER, DG CAPONE, EW BOYER, RW HOWARTH et al. (2004) Nitrogen cycles: Past, present, and future. Biogeochemistry 70: 153-226.

HABERL H, V WINIWARTER, K ANDERSSON, RU AYRES, C BOONE et al. (2006) From LTER to LTSER: Conceptualizing the socioeconomic dimension of long-term socioecological research. Ecology and Society 11: 13.

HOBBIE JE, SR CARPENTER, NB GRIMM, JR GOSZ \& TR SEASTEDT (2003) The US Long-Term Ecological Research Program. BioScience 53: 21-32.

KELLER M, DS SCHIMEL, WW HARGROVE \& FM HOFFMAN (2008) A continental strategy for the National Ecological Observatory Network. Frontiers in Ecology and the Environment 6: 282-284.

LATORRE C, PI MORENO, G VARGAS, A MALDONADO, R VILLA-MARTINEZ et al. (2007) Late Quaternary environments and palaeoclimate. En: Moreno T \& W Gibbons (eds) The geology of Chile: 309-328. The Geological Society, London, UK.

LAWFORD RG, PB ALABACK \& E FUENTES (eds) (1996) High latitude rainforests and associated ecosystems of the west coast of the Americas: Climate, hydrology, ecology and conservation. Springer-Verlag, New York.

MAASS M, R DÍAZ-DELGADO, P BALVANERA, A CASTILLO \& A MARTÍNEZ-YRÍZAR (2010) Redes de Investigación Ecológica y SocioEcológica a Largo Plazo (LTER y LTSER) en Iberoamérica: Los casos de México y España. Revista Chilena de Historia Natural 83: 171-184.

NSF (2002) Long-Term Ecological Research Program
Twenty-Year Review National Science Foundation. (en línea) URL: http:// intranet.1ternet.edu/archives/documents / reports/20_yr_review/ (accedido Enero 27, 2010).

PIELKE JR R, G PRINS, S RAYNER \& D SAREWITZ (2007) Lifting the taboo on adaptation. Nature 445: 597-598.

REDMAN CL, JM GROVE \& LH KUBY (2004) Integrating social science into the long-term ecological research (LTER) network: Social dimensions of ecological change and ecological dimension of social change. Ecosystems 7: 161171.

ROZZI R, F MASSARDO, J SILANDER JR, CB ANDERSON \& A MARIN (2003) Conservación biocultural y ética ambiental en el extremo austral de América: Oportunidades y dificultades para el bienestar ecosocial. En: Figueroa E \& J Simonetti (eds) Biodiversidad y Globalización: 51-85. Editorial Universitaria, Santiago, Chile.

ROZZI R, F MASSARDO, A BERGHÖFER, C ANDERSON, A MANSILLA et al. (2006) La Reserva de Biosfera Cabo de Hornos. Programa MaB-UNESCO. Ediciones Universidad de Magallanes, Punta Arenas.

ROZZI R, J ARMESTO \& R FRODEMAN (2008) Integrating ecological sciences and environmental ethics into biocultural conservation in South American temperate subantarctic ecosystems. Environmental Ethics 30: 229-234.

STRAYER DL, JS GLITZENSTEIN, C JONES, J KOLASA, GE LIKENS, M MCDONNEL, GG PARKER \& STA PICKETT (1986) Long-term ecological studies: An illustrated account of their design, operation and importance to ecology. Occassional Publication of the Institute of Ecosystem Studies. Millbrook, New York.

SWANSON FJ, C GOODRICH \& KD MOORE (2008) Bridging boundaries: Scientists, creative writers, and the long view of the forest. Frontiers in Ecology and the Environment 6: 499-504. 
